\title{
Oxidación y antioxidantes: hechos y controversias
}

Oxidation and antioxidants: facts and controversies

\author{
Claudia Juliana Mariaca' ${ }^{1}$, Mónica Zapata², Paulina Uribe ${ }^{3}$ \\ 1. Médica dermatóloga, Universidad Pontificia Bolivariana, Medellín, Colombia. \\ 2. Médica dermatóloga, Universidad CES, Medellín, Colombia. \\ 3. Médica dermatóloga; docente de Dermatología, Universidad Pontificia Bolivariana, Medellín, Colombia.
}

\section{RESUMEN}

El envejecimiento se caracteriza por cambios progresivos en los tejidos que dan lugar a la disminución de la función y, finalmente, a la muerte. A lo largo de los años se han descrito múltiples teorías tratando de explicar por qué se produce el envejecimiento. Una de las más aceptadas es la teoría de los radicales libres, la cual sugiere que las especies reactivas del oxígeno forman productos endógenos al reaccionar con otras moléculas, y que estas juegan un papel importante en el envejecimiento. Las células y los órganos del cuerpo tienen sistemas antioxidantes, los cuales pueden ser enzimáticos, no enzimáticos o proteínas de unión. Todos actúan sinérgicamente para neutralizar las diferentes especies reactivas del oxígeno, formando una red de antioxidantes que retrasan el proceso de envejecimiento.

PALABRAS CLAVE: radicales libres, envejecimiento, antioxidantes, piel.

\section{SUMMARY}

Aging is characterized by progressive changes in the tissues that give place to a decrease in their function and finally death. Over the years, many theories have tried to explain why aging is produced. One of the most accepted is the theory of free radicals that suggests that reactive oxygen species produce endogenous products when they react with other molecules, playing an important role in the aging process.

KEY WORDS: Free radicals, aging, antioxidants, skin.

\section{INTRODUCCIÓN}

El envejecimiento se caracteriza por cambios progresivos en los tejidos que dan lugar a disminución de la función y, finalmente, a la muerte del organismo. Se define como un proceso continuo que se inicia en la concepción y continúa hasta la muerte ${ }^{1,2}$.

En los organismos aerobios, el oxígeno es esencial para la producción de energía, pero, paradójicamente, produce estrés tóxico crónico en las células. Por lo tanto, existen mecanismos protectores para remover los productos tóxicos del oxígeno.

\section{Correspondencia:}

Claudia Juliana Mariaca

Email:

julianamariaca@hotmail.com

Recibido: 14 de junio de 2015

Aceptado: 22 de mayo de 2016

No se reportan conflictos de interés. 
Estos mecanismos se conocen como antioxidantes, los cuales son críticos en la supervivencia ${ }^{1}$.

\section{TEORÍA DE LOS RADICALES}

\section{LIBRES}

En los últimos años, se han propuesto cientos de teorías para explicar el envejecimiento. La mayoría han probado ser incorrectas y pocas han perdurado. Una de las más estudiadas y aceptadas ha sido la teoría de los radicales libres. Se encuentran raíces de esta teoría en el siglo XIX (1894), cuando Fenton descubrió que el hierro catalizaba la oxidación del ácido tartárico a peróxido de hidrógeno, pero, solo hasta 1956 Denhan Harman sugirió que los radicales libres de oxígeno formaban productos endógenos al reaccionar con otras moléculas y que estas jugaban un papel importante en el envejecimiento. Además, sugirió que la administración de compuestos fácilmente oxidables, como la cisteína, disminuiría el estrés oxidativo y el envejecimiento, y que aumentaría la expectativa de vida ${ }^{1,3}$.

Esta teoría no ha demostrado ser completamente cierta. Se sabe que el daño oxidativo aumenta con la edad. Con los años hay un aumento de las especies reactivas del oxígeno y disminución de la capacidad antioxidante. Además, se ha comprobado que las manipulaciones que aumentan la expectativa de vida reducen el daño oxidativo. Este último punto se ha comprobado con la restricción calórica, que se define como una disminución de la disponibilidad de nutrientes en ausencia de malnutrición. Los estudios iniciales se realizaron en moscas de frutas, roedores y, más recientemente, en monos Rhesus. Se ha demostrado que la restricción calórica aumenta la expectativa de vida, retarda el envejecimiento y disminuye la acumulación de especies reactivas del oxígeno, de manera proporcional a la duración de la intervención. El mecanismo exacto por el cual esta intervención aumenta la expectativa de vida no ha sido completamente elucidado; sin embargo, se ha encontrado que la restricción calórica es capaz de aumentar la expresión de genes de enzimas antioxidantes. Asimismo, puede aumentar la expresión del gen de la sirtuína 1 (SIRT-1), implicado en el aumento de la expectativa de vida ${ }^{1,4-9}$.

A pesar de que lo anterior sustenta la teoría del envejecimiento, hasta el momento, los estudios en animales son contradictorios en cuanto a si al disminuir el estrés oxidativo se aumenta la expectativa de vida. Algunos análisis con animales transgénicos han demostrado que la sobreexpresión de enzimas antioxidantes es capaz de aumentar la expectativa de vida, sin embargo, otros no. Por lo tanto, es poco probable que el envejecimiento sea explicado solo por esta teoría. Probablemente, es consecuencia de múltiples factores, y la genética y el medio ambiente juegan un papel importante $\mathrm{e}^{4,10,11}$.

\section{ESPECIES REACTIVAS DEL OXÍGENO}

Un radical libre es aquella molécula que contiene uno o más electrones no pareados que buscan capturar electrones de otras sustancias para neutralizarse. Los radicales libres comprenden los radicales libres del oxígeno, el nitrógeno, el azufre y el cloro, siendo los más abundantes los radicales libres del oxígeno, también llamadas especies reactivas del oxígeno. Los radicales libres tienen una vida media corta; sin embargo, tienen la capacidad de reaccionar rápidamente con el ADN, las proteínas y los lípidos, generando daño en estos que perduran más tiempo en el organismo ${ }^{7,12-15}$.

Las especies reactivas del oxígeno se pueden generar por mecanismos endógenos o por fuentes exógenas. Los procesos endógenos que las generan incluyen el metabolismo oxidativo, el ejercicio excesivo, los mediadores inflamatorios, como las prostaglandinas, y las reacciones enzimáticas, como la reacción de Fenton. Ejemplos de las fuentes exógenas incluyen la radiación ultravioleta (UV), la polución, los agentes fotosensibilizadores, los pesticidas y el cigarrillo. El estrés oxidativo resulta cuando la producción de especies reactivas del oxígeno excede la capacidad de las defensas antioxidantes ${ }^{2,7,16}$.

\section{Anión superóxido $\left(\mathrm{O}^{2-}\right)$}

Es uno de los radicales libres más abundantes en las células. Su principal fuente es el escape de oxígeno en la cadena transportadora de electrones en la mitocondria. Tiene la capacidad de oxidar lípidos, proteínas y ácidos nucleicos, y en ocasiones puede actuar como agente reductor al donar un electrón a un metal de transición oxidado como el hierro $\left(\mathrm{Fe}^{+2}\right)$ o el cobre $\left(\mathrm{Cu}^{+2}\right)$, como ocurre en la reacción de Fenton. Es convertido a peróxido de hidrógeno $\left(\mathrm{H}_{2} \mathrm{O}_{2}\right.$, otro radical libre) por la superóxido dismutasa ${ }^{7,12,17-20}$.

\section{Peróxido de hidrógeno ( $\left.\mathrm{H}_{2} \mathrm{O}_{2}\right)$}

Es uno de los radicales libres menos potentes, pero, cuando se produce en presencia de metales de transición como $\mathrm{Fe}^{+3}$ o $\mathrm{Cu}^{+}$, los puede oxidar y 


\section{$\mathrm{Fe}^{2+}+\mathrm{H}_{2} \mathrm{O}_{2} \longrightarrow \mathrm{Fe}(\mathrm{III})+\mathrm{OH}^{-}+\mathrm{OH}^{-}$}

FIGURA 1. Reacción de Fenton.

producir un anión férrico $\left(\mathrm{Fe}^{+2}\right)$ o cúprico $\left(\mathrm{Cu}^{+2}\right)$ en la reacción de Fenton, generando el radical hidroxilo $^{14,18}$ (FIGURA 1).

\section{Radical hidroxilo $(\cdot \mathrm{OH})$}

Es muy reactivo e inestable, interacciona con la primera molécula que entre en contacto, como por ejemplo el ADN, alterando la capacidad de división de la célula y dando lugar a mutaciones. Además, puede extraer moléculas de hidrógeno $\left(\mathrm{H}^{+}\right)$de los ácidos grasos polisaturados, creando radicales lipídicos que pueden alterar la barrera cutánea y activar mecanismos inflamatorios. Es el radical más difícil de eliminar por medio de los antioxidantes, por lo que el organismo trata de evitar su producción al bloquear la reacción de Fenton por medio de la disminución de la disponibilidad del hierro y otros metales. Se ha sugerido que las mujeres tienen mayor expectativa de vida debido a que pierden hierro cada mes con el periodo menstrual, generando menos radicales hidroxilo ${ }^{18,21-23}$.

\section{Oxígeno singlete $\left({ }^{1} \mathrm{O}_{2}\right)$}

Es una forma energéticamente excitada de oxígeno molecular y, junto con el radical hidroxilo, son los radicales libres con vida media más corta y, por lo tanto, los más reactivos. Una vez formado, reacciona rápidamente en dos formas: 1) dona un electrón al oxígeno molecular, dando como resultado $\mathrm{O}^{2-}$, y 2) extrae un átomo de $\mathrm{H}^{+}$de un ácido graso insaturado dando origen a un radical lipídico.

Debido a que el oxígeno tiene dos órbitas no pareadas, se considera un radical libre. Para el oxígeno $\left(\mathrm{O}_{2}\right)$ molecular no es diferente, por lo que se considera un birra- dical, que tiene la tendencia de unirse a dos moléculas de $\mathrm{H}^{+}$para formar agua ${ }^{21-23}$.

Una fuente significativa de especies reactivas del oxígeno proviene del metabolismo oxidativo en la mitocondria, donde el ATP es generado a partir de la glucosa en la cadena trasportadora de oxígeno. Se estima que aproximadamente del 1 al $2 \%$ del oxígeno se escapa de la cadena transportadora de electrones y es convertido en $\mathrm{O}^{2-}$, a partir del cual se generan otros radicales libres ${ }^{18,23}$.

Las especies reactivas del oxígeno por su capacidad de inducir daño en el ADN, los lípidos y las proteínas, pueden alterar la función de las estructuras biológicas, por lo que se han implicado en la enfermedad de Alzheimer, la de Parkinson, el accidente cerebrovascular, la enfermedad cardiovascular, la artritis, las cataratas y el envejecimiento ${ }^{18,23,24}$.

Los radicales libres producen alteraciones en diferentes tejidos y la piel no está exenta de esto. Debido a que la piel está expuesta al oxígeno, no es sorprendente que sea vulnerable al estrés inflamatorio y, por ser el órgano del cuerpo más externo, frecuentemente está expuesta a un ambiente pro-oxidativo como la radiación UV, la radiación infrarroja y la polución. La radiación UV, especialmente en el espectro UVA, es un potente iniciador de estrés oxidativo en la piel y es la fuente más importante de radicales libres, generando daño en el ADN, las proteínas y los lípidos. La oxidación de las proteínas produce daño en estas, se acumulan y persisten principalmente en la dermis, induciendo la producción de metaloproteinasas (matrix metalloproteinase, MMP) con la subsecuente degradación de la matriz ${ }^{18,25,26}$.

El daño de la piel por los radicales libres juega un papel muy importante en el fotoenvejecimiento. Después de la exposición a la radiación UV, las especies 


\begin{tabular}{cc}
\hline HIDROSOLUBLES & LIPOSOLUBLES \\
\hline Ácido ascórbico & Tocoferol \\
Glutatión & Ubicuinol \\
Cisteína & Retinoides \\
Ácido lipoico & Carotenos \\
Ácido úrico & \\
\hline
\end{tabular}

TABLA 1. Antioxidantes no enzimáticos.

reactivas del oxígeno estimulan la liberación de citocinas proinflamatorias y factores de crecimiento, específicamente, el factor activador de proteína 1 (AP-1) y el factor nuclear B (NF-B); estos producen una regulación positiva de las MMP, como las MMP 1, 3, 8 y 9. Estas proteasas degradan el colágeno y las fibras de elastina de la matriz extracelular. Se ha confirmado, además, que los radicales libres disminuyen la expresión del factor de crecimiento beta, con lo que se reduce la producción de colágeno y aumenta su degradación ${ }^{27-29}$.

En los melanocitos, los radicales libres tienen una acción paradójica; no solo aumentan la pigmentación, sino que también pueden inducir pérdida del pigmento. Un ejemplo de la degeneración de los melanocitos inducida por el estrés oxidativo es el vitiligo. Los pacientes con vitiligo tienen mayor cantidad de superóxido dismutasa en la piel y bajos niveles de catalasa, lo que resulta en un desequilibrio, por lo que aumenta la producción de $\mathrm{H}_{2} \mathrm{O}_{2}$ que se acumula en la piel ${ }^{24-26,30}$.

Es importante recalcar que, a pesar de que las especies reactivas del oxígeno pueden causar daño, también juegan un papel importante en la homeostasis celular. Por ejemplo, actúan como mensajeros intracelulares, con un papel sustancial en las señales celulares; son reguladores de la producción de citocinas, regulan el crecimiento celular, y son producidas por los macrófagos para ayudar en el proceso de eliminación de microorganismos. Por lo tanto, se necesitan pequeñas cantidades para el funcionamiento adecuado de los tejidos ${ }^{18,26,30,31}$.

\section{ANTIOXIDANTES}

Por definición, un antioxidante es cualquier sustancia que retrasa o previene la oxidación de un sustrato oxidable a pesar de estar presente en concentraciones

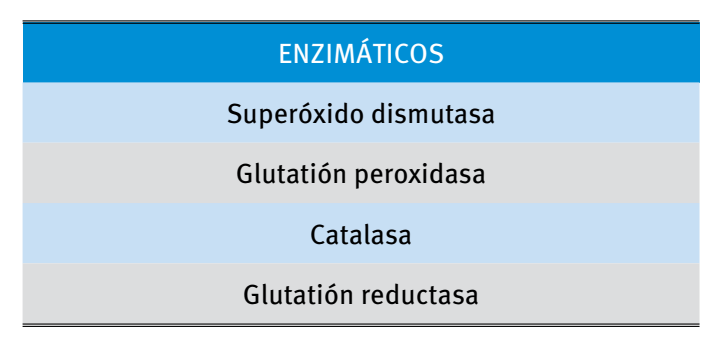

TABLA 2. Antioxidantes enzimáticos.

más bajas que el sustrato. Las células y los órganos del cuerpo tienen sistemas antioxidantes, los cuales pueden ser enzimáticos, no enzimáticos o proteínas de unión. Todos actúan sinérgicamente para neutralizar las diferentes especies reactivas del oxígeno, formando una red de antioxidantes (TABLAS 1 Y 2) $)^{18,26,32}$.

Igualmente, la piel está equipada con una red de antioxidantes protectores enzimáticos, que son los más importantes y son la primera barrera contra los radicales libres, como la glutatión peroxidasa, la superóxido dismutasa y la catalasa; también, con antioxidantes de bajo peso molecular no enzimáticos, como la vitamina E, la vitamina C, el glutatión, el ácido úrico y los carotenoides. En general, la epidermis contiene mayor concentración de antioxidantes que la dermis, por lo que su capacidad antioxidante es mayor. Es interesante que hay mayor concentración de antioxidantes en las capas más profundas de la epidermis; esto podría explicarse porque la presión parcial de $\mathrm{O}_{2}$ es mayor en las capas superficiales, lo que causa estrés oxidativo y da lugar a la disminución de los antioxidantes (FIGURA 2) 25,26,32-34.

\section{Antioxidantes no enzimáticos}

Vitamina C o ácido L-ascórbico. Debido a que no se ha podido sintetizar artificialmente, se debe obtener de la dieta, principalmente de frutas cítricas, tomates y otros vegetales. Es el antioxidante predominante en la piel con base en las concentraciones molares. Es hidrosoluble, por lo cual neutraliza los radicales libres en los compartimentos acuosos de la piel. Elimina la mayoría de las especies reactivas del oxígeno por la oxidación del ascorbato a monodihidroascorbato y, posteriormente, a dihidroascorbato. Además de servir como antioxidante, es un cofactor de varias enzimas para la sintesis de colágeno y ayuda a reciclar la vitamina E. Para mantener los niveles adecuados de vitamina $\mathrm{C}$ re- 


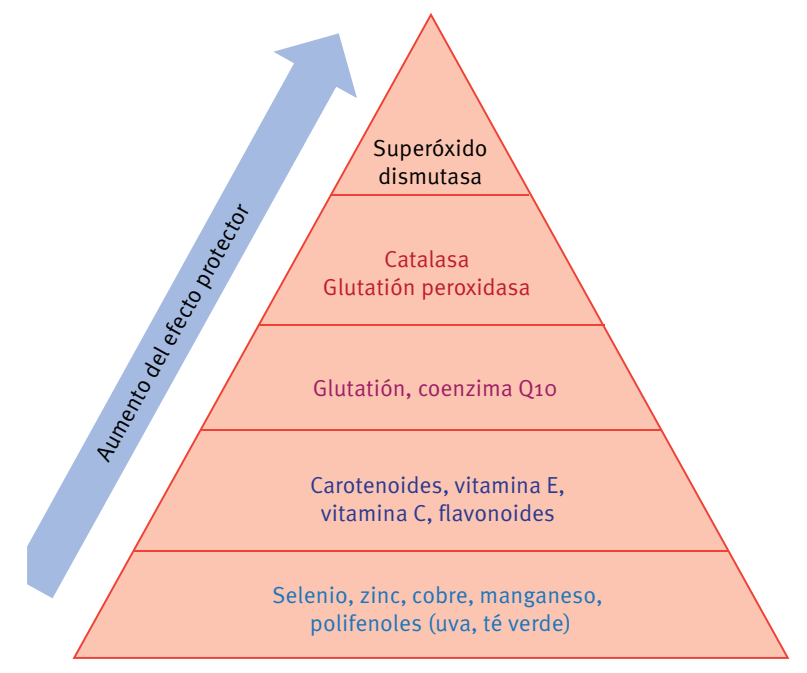

FIGURA 2. Jerarquía de los antioxidantes. ducida, las células generan tioles como el glutatión, el cual reduce el radical semiascorbil (forma oxidada) a ascorbato (forma reducida) 33,35 .

La vitamina $\mathrm{C}$ se absorbe en el intestino delgado distal cuando se ingiere una dosis de $100 \mathrm{mg}$ al día; cuando las dosis son mayores, se absorbe solo una fracción.

Se ha encontrado una relación inversa entre los niveles plasmáticos de ácido ascórbico y los de isoprostanos. Estos son una familia de prostaglandinas que hacen parte de la vía de la inflamación no enzimática. La medición de su concentración en orina provee información útil del daño oxidativo y representa un biomarcador excelente de la peroxidación lipídica. Se ha demostrado que la expresión aumentada de neuroprostanos está implicada en la enfermedad de Alzheimer. En un estudio doble ciego con placebo, la administración de vitamina C, $500 \mathrm{mg}$ dos veces al día, disminuyó los niveles urinarios de metabolitos de peroxidación lipídica en 20 a 30 \%, lo cual sustenta que el suplemento de vitamina $\mathrm{C}$ tiene efecto antioxidante in vivo. Se debe tener en cuenta, sin embargo, que la administración oral de esta vitamina tiene un efecto dual que puede ser antioxidante o pro-oxidante, dependiendo de la dosis. Si se administran dosis altas (aún no establecidas), actúa como pro-oxidante y facilita la reacción de Fenton ${ }^{18,25,30,33}$.

En cuanto a su aplicación tópica, cabe recalcar que el ácido ascórbico es inestable de manera innata y no se sabe si permanece intacto en la piel después de su aplicación. Por esta razón, se ha modificado químicamente al esterificar su grupo hidroxilo para producir derivados como el palmitato de ascorbilo, el tetraisopalmitato de ascorbilo y el fosfato magnesio de ascorbilo. Un estudio reveló que el pH óptimo debe ser menor de 3,5 y que la concentración máxima para una absorción óptima es de $20 \% 0^{36,37}$.

Vitamina E. Es un antioxidante liposoluble que se obtiene de la dieta a partir de vegetales como la espinaca, de aceites vegetales, del germen de trigo y de las nueces. Existe en ocho componentes mayores, cuatro tocoferoles (alfa, beta, gamma y delta) y cuatro tocotrienoles; el alfa-tocoferol es la forma más potente y abundante 37,38 .

$\mathrm{Su}$ efecto antioxidante se debe en parte a su grupo hidroxilo, el cual dona el $\mathrm{H}^{+}$para neutralizar los radicales libres. Su principal función es proteger las membranas celulares del estrés oxidativo, actuando como primera barrera contra la peroxidación lipídica. Sus propiedades antioxidantes dependen de su regeneración continua por otros agentes biológicos. El ácido ascórbico y el glutatión son esenciales para mantener la acción de la vitamina $\mathrm{E}$, ya que estos donan iones de $\mathrm{H}^{+}$cuando se forma el radical tocoferol. Además, el selenio actúa como cofactor en la regeneración de la vitamina $E$, por lo que la deficiencia de selenio puede simular la deficiencia de esta vitamina ${ }^{37}$. En la piel, el alfa-tocoferol se encuentra principalmente en el estrato córneo, con mayor concentración en sus capas más profundas. La radiación de la piel a dosis más 
bajas que la dosis mínima de eritema reduce el alfatocoferol en el estrato córneo en $50 \% \%^{37}$.

En un estudio de asignación aleatoria con 400 UI de vitamina E por 14 días, se evaluó su aumento en la piel. Los niveles de alfa-tocoferol al día 21 aumentaron de 87 a $92 \%$ en la secreción sebácea (no hubo cambio en el día 14). Este estudio sugiere que la secreción de la glándula sebácea es un mecanismo de entrega de alfatocoferol a la piel y que, para que haya un aumento significativo, se requiere un suplemento diario por un período mínimo de dos a tres semanas ${ }^{37,39,40}$.

No se ha documentado toxicidad, pero suplementos orales a dosis mayores a 1.000 UI día han mostrado efecto pro-oxidante, además de que pueden tener un efecto negativo en la agregación plaquetaria y pueden interactuar con el metabolismo de otros medicamentos, ya que esta vitamina es metabolizada por el sistema citocromo P-450 $\mathrm{O}^{25,36,37}$.

Se ha demostrado que, cuando la piel es expuesta a radiación ultravioleta $B$ (UVB) y la vitamina E es aplicada tópicamente a concentraciones de o,1 a $1 \%$ antes 0 después de la exposición, se pueden suprimir el edema, el eritema, la peroxidación de lípidos, el fotoenvejecimiento, la fotocarcinogénesis y la melanogénesis. Con la combinación de ácido ascórbico al 15 \% se duplica la protección; además, con la adición de agentes estabilizantes, como el ácido ferúlico al 1,5\% y la floretina, se produce un mayor beneficio. La vitamina $\mathrm{C}$ debe ser incluida en cualquier formulación tópica que contenga vitamina $\mathrm{E}$, no solo porque contribuye a su acción al reciclarla, sino también, porque ayuda a estabilizarla para evitar su degradación por la radiación ultravioleta A (UVA). A diferencia de la vitamina E oral, la aplicación tópica de vitamina $\mathrm{C}$ es segura; los efectos secundarios se limitan a irritación leve $e^{24,26,33,41,42}$.

Carotenos. Son pigmentos orgánicos que se producen naturalmente en las plantas, las algas, algunos tipos de hongos y algunas bacterias. Los carotenoides más comunes son el beta-caroteno, el alfa-caroteno y la beta-criptoxantina. Se consideran antioxidantes porque tienen la habilidad de mitigar el ${ }^{1} \mathrm{O}_{2}$ y los radicales lipídicos. Además, se ha confirmado que pueden proteger contra el daño inducido por la radiación UV, y que ayudan a prevenir la degradación del colágeno. $\mathrm{Su}$ efectividad en la fotoprotección sistémica depende de la duración del tratamiento. Para que sea efectivo, se debe usar, por lo menos, durante 10 semanas $^{25,37,43}$.

Coenzima Q (CoQ10). Trabaja junto con el alfa-tocoferol y está presente en la mayoría de las organelas celulares. Se puede obtener de la dieta a partir del pes- cado y de la carne o puede sintetizarse de novo. Se cree que juega un papel importante en la fotoprotección endógena, ya que su concentración en la piel se disminuye después de la radiación UV. Además, disminuye el daño del ADN causado por la radiación ultravioleta A (UVA), reduce las especies reactivas del oxígeno protegiendo contra el estrés oxidativo y suprime la producción de MMP-1 en los fibroblastos ${ }^{25}$.

Polifenoles. Son potentes antioxidantes con la capacidad de eliminar los radicales superóxido, radicales lipídicos $\mathrm{y}^{1} \mathrm{O}_{2}$; además, son capaces de estimular la producción de enzimas antioxidantes como la superóxido dismutasa ${ }^{34}$. Entre los polifenoles se encuentran el té verde y el resveratrol.

Té verde. Es la bebida más popular después del agua. Es rico en polifenoles: epicatequina (EC), epicatequina galeato (ECG), epigalocatequina (EGC) y epigalocatequina 3 galeato (EGCG). Esta última es el más abundante y ha demostrado una potencia de 25 a 100 veces mayor que las de las vitaminas $\mathrm{C}$ y E. El té verde ha mostrado efectos positivos contra la inflamación, el estrés oxidativo y el daño del ADN. Numerosos estudios in vivo e in vitro han probado que su suplemento oral o su aplicación tópica previenen la fotocarcinogénesis, evitan la disminución de los antioxidantes en la epidermis, repara el daño inducido por la radiación UVB y aumenta la expresión del gen supresor $p 53$. Sin embargo, algunos estudios han evidenciado un efecto pro-oxidante, lo cual sugiere que tiene una acción dual dependiendo de la dosis, de la duración de la administración y de la interacción con otros componentes de la dieta. Los estudios en humanos son limitados, por lo que no se ha establecido el rango seguro de su consumo. Con base en la información obtenida de estudios animales, se puede sugerir que el consumo regular de cinco a seis tazas de té verde al día tiene un efecto antioxidante ${ }^{25,34,44,45}$.

Resveratrol. Es otro polifenol encontrado en la piel y en las semillas de las uvas, las nueces, las frutas y el vino rojo. Es uno de los componentes reportados como más potentes antioxidantes. Es capaz de prevenir el envejecimiento cutáneo inducido por la radiación UV, de inhibir la tirosinasa, y tiene actividad antiinflamatoria y antiproliferativa. Se ha encontrado que reduce la concentración intracelular de $\mathrm{H}_{2} \mathrm{O}_{2}$ y elimina el radical hidroxilo.

En los últimos años se ha reportado que el resveratrol protege la piel contra el daño oxidativo al aumentar la expresión de genes antioxidantes, como el factor de transcripción Nrf2 (NFE2-related factor 2) y la proteína KEAP1 (Kelch-like ECH-associated protein 1), que se 


\section{"Los polifenoles son potentes antioxidantes con la capacidad de eliminar los radicales superóxido, radicales lipídicos y 102; además, son capaces de estimular la producción de enzimas antioxidantes como la superóxido dismutasa"}

consideran proteínas esenciales en la coordinación de la transcripción de varias enzimas antioxidantes. En condiciones fisiológicas, el Nrf2 está unido a la Keap1 y son retenidos en el citoplasma. Hay inductores que los separan, causando la traslocación del Nrf2 al núcleo y se una al elemento de reacción antioxidante (ARE), induciendo la expresión de enzimas antioxidantes. Los estudios con resveratrol han demostrado que su efecto antioxidante se debe, en parte, a que es capaz de activar el factor Nrf2 y acelerar su traslocación al núcleo. En un estudio con cultivos de queratinocitos humanos, el tratamiento con resveratrol antes de la exposición a radiación UV disminuyó la producción de especies reactivas del oxígeno en los queratinocitos $34,44,46$. Además, se ha reportado que el resveratrol puede activar la sirtuína 1, lo cual sugiere que podría simular la restricción calórica con el consecuente incremento de la expectativa de vida, sin necesidad de someter a los pacientes a la restricción de calorías ${ }^{47}$.

Glutatión. Es un tripéptido endógeno y es el tiol más abundante en la mayoría de los tejidos. Es una importante defensa contra el daño oxidativo. Se sintetiza en el hígado y es transportado a los tejidos. Cuando se ingiere, es degradado en el intestino delgado, por lo que los niveles celulares no están influenciados por la dieta. Tiene la capacidad de reducir la vitamina $\mathrm{C}$, y junto con esta, reducen la vitamina E. Mitiga la acción de los radicales al donar un átomo de $\mathrm{H}^{+}$formando disulfito de glutatión; el glutatión reducido (GSSG) es convertido nuevamente en glutatión por la glutatión reductasa, cuando el NADPH (nicotinamide adenine dinucleotide phosphate) dona el $\mathrm{H}^{+}$. El glutatión se forma por la gamma-glutamil sintetasa y uno de sus sustratos es la cisteína, por lo que los derivados de esta se pueden usar para aumentar la producción de glutatión ${ }^{18,40}$.
Ácido lipoico. Es un tiol endógeno hidrosoluble y liposoluble que sirve como cofactor en complejos de deshidrogenasas en la mitocondria, por lo que juega un papel importante en el metabolismo energético. Puede ser consumido en la dieta (vegetales de hoja verde, carnes, hígado, riñón) o ser sintetizado de novo a partir del ácido octanoico. Es convertido a su forma reducida, el ácido dihidrolipoico (DHLA), el cual es un potente antioxidante. Debido a que la pareja ácido lipoico más ácido dihidrolipoico (AL/DHLA) posee actividad antioxidante, esto lo hace un antioxidante único, ya que su forma oxidada y la reducida poseen efectos antioxidantes.

Los suplementos en la dieta contienen entre $50 \mathrm{y}$ $600 \mathrm{mg}$, y su absorción es de 20 a $40 \%$, la cual se disminuye con la comida. Los niveles plasmáticos son máximos en 0,5 a 2 horas después de su administración. Se metaboliza rápidamente y se excreta por la orina, por lo que su acumulación en los tejidos es escasa después de su ingestión. Por lo tanto, se cree que actúa indirectamente al aumentar la capacidad antioxidante de las células. Se ha demostrado que aumenta los niveles de glutatión al activar los genes de transcripción mediante el factor Nrf2. Además, es capaz de aumentar la captación de cisteína, un importante cofactor en la síntesis de glutatión; también, es capaz de quelar metales como el cobre, el hierro y el cinc, previniendo la reacción de Fenton y evitando la formación del radical hidroxilo. Asimismo, puede regenerar otros antioxidantes como las vitaminas $\mathrm{C}$ y E. No se ha documentado toxicidad con su suplemento. En algunos estudios, con la administración de 2.400 $\mathrm{mg} /$ día no se reportaron efectos secundarios cuando fue comparado con el placebo ${ }^{48-50}$.

La mayoría de los estudios en humanos se han realizado en pacientes con diabetes mellitus. En Alemania 
se ha usado por más de 30 años en la neuropatía diabética y ha demostrado que mejora la captación de la glucosa y la tolerancia a esta; en el músculo, aumenta la expresión del GLUT4 (glucose transporter type 4). Además, se ha confirmado que es muy afín al tejido nervioso y se ha usado en el herpes zóster en el tratamiento y la prevención de la neuralgia posherpética ${ }^{51-53}$.

\section{Antioxidantes enzimáticos}

Superóxido dismutasa. Junto con la glutatión peroxidasa, es una de las enzimas más activas. Son proteínas que contienen metales que catalizan la remoción de $\mathrm{O}^{2-}$, generando $\mathrm{H}_{2} \mathrm{O}_{2}$ como producto final. Hay tres isoformas: la superóxido dismutasa 1, localizada a nivel intracelular que usa el cobre y el cinc como cofactores; la superóxido dismutasa 2, localizada en la mitocondria que usa el magnesio como cofactor, y la superóxido dismutasa 3, extracelular, que usa el cobre y el cinc como cofactores. La superóxido dismutasa 2 se ha encontrado en la piel y se sabe que la exposición repetitiva a la radiación UVA aumenta su producción. En la piel también es capaz de proteger contra la fragmentación del colágeno de tipo I y de inhibir la activación de colagenasas; además, tiene propiedades antiinflamatorias y disminuye el daño producido por la radiación UV $\mathrm{V}^{5,18,38,40}$.

Catalasa. Se localiza principalmente en los peroxisomas y, en pequeñas cantidades, en la mitocondria. Es una dismutasa, por lo que no necesita cofactores. Elimina el $\mathrm{H}_{2} \mathrm{O}_{2}$ en la piel y su actividad se disminuye después de la exposición a radiación UVA y UVB ${ }^{18,43,54,55}$.

Glutatión peroxidasa. Es uno de los antioxidantes celulares más abundantes y se considera la enzima antioxidante más importante en la piel. Es una reductasa que necesita NADPH para catalizar su reacción, y se localiza en el citoplasma y en la matriz mitocondrial. Al igual que la catalasa, elimina el $\mathrm{H}_{2} \mathrm{O}_{2}$ en presencia de glutatión reducido y además, limita la peroxidación de lípidos. Su síntesis de novo se produce principalmente en el hígado (90 \%). En cultivos, usando células de piel humana, se ha demostrado que la disminución de esta enzima aumenta la sensibilidad a la radiación UVA y a la UVB, y que hay una correlación directa entre su concentración y el grado de sensibilización. El selenio es un agente esencial para optimizar la actividad de la enzima, ya que sus sitios activos contienen selenio. Es por eso que, en la actualidad, se usa frecuentemente en preparaciones tópicas en la forma de L-seleniometionina y ha demostrado que puede aumentar la dosis mínima de eritema. Disminuye el eritema y la quema- dura solar después de la exposición a la radiación UVB cuando se administra por vía oral, lo que indica un efecto fotoprotector ${ }^{18,43,54,55}$.

\section{Otros antioxidantes}

- Vitaminas: nicotinamida, niacinamida y riboflavina.

- Ácidos grasos: omega 3 y 6.

- Aminoácidos: arginina, histidina y glicina.

- Proteínas: albúmina, lactoferrina, transferrina, bilirrubina y ceruloplasmina.

- Minerales: cinc, hierro, cobre, selenio y cromo

- Metabolitos: ácido úrico.

- Otros sistemas enzimáticos: glutatión reductasa (regenera el glutatión reducido) y sistema de la tiorredoxina.

\section{SUPLEMENTOS ORALES EN DERMATOLOGÍA EN EL TRATAMIENTO DEL FOTOENVEJECIMIENTO}

\section{Retinoides orales}

Según diversas evaluaciones, en contraste con los retinoides tópicos, los efectos de los retinoides orales en el fotoenvejecimiento son mínimos. En tres estudios pequeños se evaluaron los retinoides orales en el envejecimiento cutáneo. En el primero, se evaluó si la adición de 10 a $20 \mathrm{mg}$ de isotretinoína oral tres veces a la semana a otras intervenciones antiedad (quirúrgicas y no quirúrgicas) tendría algún beneficio. A pesar de que los autores reportaron un beneficio, no se hizo un control con placebo. En el segundo, se encontró que, después de administrar $20 \mathrm{mg}$ de isotretinoína tres veces a la semana durante tres meses, 20 de los 30 pacientes reportaron mejoría clínica con medición objetiva histológica. En el tercero, se comparó el efecto de $10 \mathrm{mg}$ con el de $20 \mathrm{mg}$, diarios, de isotretinoína en dos grupos separados, cada uno de 15 pacientes. En ambos grupos se encontró un aumento estadísticamente significativo en el colágeno dérmico y hubo una mejoría subjetiva de la apariencia de la piel ${ }^{56-58}$.

\section{Carotenos}

No tienen un papel bien establecido en el tratamiento del fotoenvejecimiento, aunque pueden ser importantes en su prevención. En estudios in vitro se ha de- 


\section{"Según diversas evaluaciones, en contraste con los retinoides tópicos, los efectos de los retinoides orales en el fotoenvejecimiento son mínimos"}

mostrado la habilidad de los carotenos para proteger los fibroblastos contra la radiación UV. Con múltiples estudios en humanos se ha tratado de determinar si el suplemento oral con beta-caroteno es capaz de aumentar la dosis mínima de eritema y los resultados han variado. En los estudios de corta duración (menos de ocho semanas) no se ha demostrado un aumento en la dosis mínima de eritema. En otros estudios, en los cuales se ha suministrado beta-caroteno por 12 a 24 semanas, se observó un aumento significativo de dicha dosis en el tiempo, que fue mucho mayor al administrarlo concomitantemente con vitamina E. Estos hallazgos sugieren que los niveles de carotenos aumentan en la piel si se suministran, por lo menos, por 12 semanas, por lo cual su efecto protector solo se puede observar después de alcanzar ciertas concentraciones cutáneas ${ }^{59-63}$.

\section{Vitamina C}

Existen múltiples estudios que sustentan el efecto fotoprotector de la aplicación tópica en la mañana. Sin embargo, no hay evaluaciones sobre el efecto de la administración oral de esta vitamina en el fotoenvejecimiento ${ }^{37}$.

\section{Vitamina E}

Debido al importante papel que desempeña el ácido ascórbico en mantener la vitamina E activa, en algunos estudios se ha evaluado el efecto combinado de estas dos vitaminas. En un estudio de asignación aleatoria controlado con placebo, se evaluó el efecto de $2 \mathrm{~g}$ diarios de vitamina $\mathrm{C}$ y 1.000 UI diarios de alfa-tocoferol $1.000 \mathrm{UI}$ en 20 pacientes. Diez personas recibieron las vitaminas, mientras que las otras diez recibieron placebo. La dosis mínima de eritema se midió en los días o y 8. En el día 8 hubo un aumento significativo de dicha dosis en el grupo que recibió las vitaminas. En otro estudio controlado con placebo, se evaluó el efecto en la dosis mínima de eritema de $3 \mathrm{~g}$ diarios de vitamina $\mathrm{C}$, de $2 \mathrm{~g}$ diarios de vitamina $\mathrm{E}$ y de la combinación de ambas. No hubo diferencia significativa en la dosis cuando se usaron las vitaminas como monoterapia después de 50 días; sin embargo, en el grupo que recibió ambas vitaminas hubo un aumento del $78 \%$ en la dosis mínima de eritema ${ }^{64,65}$.

\section{Polypodium leucotomos (Fernblock)}

Es un helecho tropical, disponible comercialmente para uso oral o tópico; ninguna de las dos presentaciones ha presentado efectos secundarios ni toxicidad sistémica. Su actividad fotoprotectora se debe a sus propiedades antioxidantes, ya que contiene fenoles, principalmente benzoatos cinamatos, ácido cafeico y ferúlico que tienen la capacidad de eliminar radicales libres como el $\mathrm{O}^{2-},{ }^{1} \mathrm{O}_{2}$, ${ }^{\circ} \mathrm{OH}$, y $\mathrm{H}_{2} \mathrm{O}_{2}$, y además, es capaz de prevenir la peroxidación lipídica, inhibir la mutagénesis del ADN y aumentar la expresión de inhibidores de las MMP. La dosis oral que confiere efecto fotoprotector es de $7,5 \mathrm{mg} / \mathrm{kg}^{66-68}$.

\section{Glisodin $®$.}

Es una fórmula vegetal que contiene extracto de melón rico en superóxido dismutasa junto con una molécula de gliadina, una proteína extraída del trigo. Es el primer componente que se logra patentar como suplemento de superóxido dismutasa, ya que intentos anteriores fallaban debido a que la superóxido dismutasa era degradada por los ácidos del estómago. La adición de la gliadina protege la superóxido dismutasa durante su paso por el tubo digestivo, lo cual permite que se libere progresivamente sin que sea degradada. 
En estudios en animales se ha demostrado que este suplemento aumenta la actividad de la catalasa y de la glutatión peroxidasa en las células, y se ha demostrado que aumenta el F2 isoprostano. En humanos también se ha comprobado su efecto antioxidante. Es capaz de aumentar otros antioxidantes endógenos y disminuir el daño del ADN inducido por el oxígeno hiperbárico. Además, tiene propiedades antiinflamatorias y fotoprotectoras, al aumentar la dosis mínima de eritema. Cabe recordar que la superóxido dismutasa es la principal enzima antioxidante, que elimina el $\mathrm{O}^{2-}$, produciendo $\mathrm{H}_{2} \mathrm{O}_{2}$ y agua. El aumento en $\mathrm{H}_{2} \mathrm{O}_{2}$ estimula la producción de la catalasa y la glutatión peroxidasa, por lo tanto, la administración de SOD aumenta indirectamente estas dos enzimas ${ }^{69-71}$.

\section{CONCLUSIÓN}

A lo largo de los años se ha tratado de explicar el mecanismo del envejecimiento, por lo cual han surgido diferentes teorías tratando de explicarlo. Una de las más estudiadas y aceptadas hasta la fecha es la de los radicales libres. Existe evidencia suficiente de que el aumento en las concentraciones de las especies reactivas del oxígeno contribuye al envejecimiento; sin embargo, esta teoría no ha demostrado ser totalmente cierta, por lo que en la actualidad se acepta que el envejecimiento ocurre por una combinación de factores en los que se requiere tener en cuenta la genética y el ambiente en el cual se desenvuelve cada persona. No se debe olvidar que ciertos niveles de radicales libres son benéficos, por lo que no se puede pretender eliminarlos por completo.

Es importante tener presente que la radiación UV es una de las principales fuentes de especies reactivas del oxígeno en la piel, por lo que en los últimos años se han estudiado múltiples tratamientos tópicos y suplementos orales para la prevención o el tratamiento del fotoenvejecimiento. A pesar de que hasta el momento no hay muchos estudios en humanos sobre el uso de suplementos orales y que no se ha determinado la dosis óptima y segura para evitar los efectos pro-oxidantes o efectos secundarios, algunos resultados preliminares muestran efectos prometedores que, junto con una fotoprotección estricta, ayudarían en el tratamiento o en la prevención del fotoenvejecimiento.

\section{Agradecimientos}

A John Harvey Gaviria Calderón por su asesoría y acompañamiento.

\section{REFERENCIAS}

1. Bokov A, Chaudhuri A, Richardson A. The role of oxidative damage and stress in aging. Mech Aging Dev. 2004;125:811-26.

2. Polef TG, Meyer TA, Aguin PP, Bianchini RJ. Cutaneous oxidative stress. J Cosmet Dermatol. 2012;11:55-64.

3. Harman D. Aging: A theory based on free radical and radiation chemistry. J Gerontol. 1956;11:298-300.

4. Muller FL, Lustgarten MS, Jang Y, Richardson A, van Remmen H. Trends in oxidative aging theories. Free Radic Biol Med. 2007;43:477-503.

5. Salmon AB, Richardson A, Pérez VI. Update on the oxidative stress theory of aging: Does oxidative stress play a role in aging or healthy aging? Free Radic Biol Med. 2010;48:642-55.

6. Sohal RS, Mocket RJ, Orr WC. Mechanisms of aging: An appraisal of the oxidative stress hypothesis. Free Radic Biol Med. 2002;33:575-86.

7. Balcombe NR, Sinclair A. Ageing: Definitions, mechanisms and the magnitude of the problem. Best Pract Res Clin Gastroenterol. 2001;15:835-49.

8. Colman RJ, Beasley TM, Kemnitz JW, Johnson SC, Weindruch $\mathrm{R}$, Anderson RM. Caloric restriction reduces age-related and all-cause mortality in Rhesus monkeys. Nat Commun. 2014;5:3557.

9. Walsh ME, Shi Y, van Remmen H. The effects of dietary restriction on oxidative stress in rodents. Free Radic Biol Med. 2014;66:88-99.

10. Zhang Y, Ikeno Y, Qi W, Chauduri A, Li Y, Bokov A, et al. Mice deficient in both Mn superoxide dismutase and glutathione peroxidase- 1 have increased oxidative damage and a greater incidence of pathology but no reduction in longevity. J Gerontol A Biol Sci Med Sci. 2009;64:1212-20.

11. Sohal RS, Orr WC. The redox stress hypothesis of aging. Free Radic Biol Med. 2012;52:539-55.

12. Halliwell B. Free radicals and antioxidants: Updating a personal view. Nutr Rev. 2012;70:257-65.

13. Cesari M, Vellas B, Gambassi G. The stress of aging. 2013;48:451-6.

14. Halliwell B. Free radicals and antioxidants: A personal view. Nutr Rev. 2004;52:253-65.

15. Niki E. Assessment of antioxidant capacity in vitro and in vivo. Free Radic Biol Med. 2010;49:503-15.

16. Gutteridge JMC, Halliwell B. Antioxidants: Molecules, medicines and myths. Biochem Biophys Res Commun. 2010;393:561-4.

17. Benzie IF. Evolution of dietary antioxidants. Comp Bioch Physiol. 2003;136:113-26.

18. Polefka TG, Meyer TA, Agin PP, Bianchini RJ. Cutaneous oxidative stress. J Cosmet Dermatol. 2012;11:55-64.

19. Sies H. Strategies of antioxidant defense. Eur J Biochem. 1993;215:213-9.

20. Halliwell B. Reactive species and antioxidants. Redox biology is a fundamental theme of aerobic life. Plant Physiol 2006;141:312-22.

21. Lu T, Finkel T. Free radical and senescence. Exp Cell Res. 2088;314:1918-22.

22. Sohal RS, Orr WC. The redox stress hypothesis of aging. Free Radic Biol Med. 2012;52:539-55.

23. Cornelli U. Antioxidant use in nutraceuticals. Clin Dermatol. 2009;27:175-94. 
24. Bezie IF. Evolution of dietary antioxidants. Comp Biochem Physiol A Mol Integr Physiol. 2003;136:113-26.

25. Masaki H. Role of antioxidants in the skin: Anti-aging effects. J Dermatol Sci. 2010;58:85-90.

26. Chen L, Hu JY, Wang SQ. The role of antioxidants in photoprotection: A critical review. J Am Acad Dermatol. 2012;67:1013-24.

27. Yaar M, Gilchrest BA. Photoaging: Mechanism, prevention, and therapy. Br J Dermatol. 2007;157:874-87.

28. Scharffetter-Kochanek K, Wlaschek M, Brenneisen $P$, Schauen M, Blaudschun R, Wenk J. UV-induced reactive oxygen species in photocarcinogenesis and photoaging. Biol Chem. 1997;378:1247-57.

29. Fisher GJ, Kang S, Varani J, Bata-Csorgo Z, Wan Y, Datta S, et al. Mechanisms of photoaging and chronological skin aging. Arch Dermatol. 2002;138:1462-70.

30. Fusco D, Colloca G, Lo Monaco MR, Cesari M. Effects of antioxidants on the aging process. Clin Interv Aging. 2007;2:377-87.

31. Hancock JT, Desikan R, Neill SJ. Role of reactive oxygen species in cell signaling pathways. Biochem Soc Trans. 2001;39:(Pt.2):342-50.

32. Kaliora AC, Dedoussis GV, Schmidt H. Dietary antioxidants in preventing atherogenesis. Atherosclerosis. 2006;187:1-17.

33. Junkins-Hopkins JM. Antioxidants and their chemopreventive properties in dermatology. J Am Acad Dermatol. 2010;62:663-5.

34. Lorencini M, Brohem CA, Dieamant GC, Zanchin NI, Maibach HI. Active ingredients against human epidermal aging. Ageing Res Rev. 2014;15:100-15

35. Arrigoni O, De Tulio MC. Ascorbic acid: Much more than just an antioxidant. Biochim Biophys Acta. 2002;1569:1-9.

36. Zussman J, Ahdout J, Kim J. Vitamins and photoaging: Do scientific data support their use? J Am Acad Dermatol. 2010;63:507-25.

37. Pinnel SR, Yang H, Omar M, Monteiro-Riviere N, DeBuys HV, Walker LC, et al. Topical L-ascorbic acid: Percutaneous absorption studies. Dermatol Surg. 2001;27:137-42.

38. Thiele JJ, Hsieh SN, Ekanayake-Mudiyanselage S. Vitamin E: Critical review of its current use in cosmetic and clinical dermatology. Dermatol Surg. 2005;31:805-13.

39. Limón-Pacheco J, Gonsebatt ME. The role of antioxidants and antioxidant-related enzymes in protective responses to environmentally induced oxidative stress. Mutat Res. 2009;674;137-47.

40. Chaudiére J, Ferrari-lliou R. Intracellular antioxidants: From chemical to biochemical mechanisms. Food Chem Toxicol. 1999;37:949-62.

41. Thiele JJ, Ekanayake-Mudiyanselage S. Vitamin E in human skin: Organ-specific physiology and considerations for its use in dermatology. Mol Aspects Med. 2007;28:646-67.

42. Traber MG, Stevens JF. Vitamin C and E: Beneficial effects from a mechanistic perspective. Free Radic Biolo Med. 2011;51:1000-13.

43. Steenvoorden DP, van Henegouwen GM. The use of endog enous antioxidants to improve photoprotection. J Photochem Photobiol B. 1997;41:1-10.

44. Braicu C, Ladomery MR, Chedea VS, Irimie A, BerindanNeagoe I. The relationship between the structure and biological actions of green tea catechins. Food Chem. 2013;141:3282-9.
45. Afag F, Mukhtar H. Biotanical antioxidants in the prevention of photocarcinogenesis and photoaging. Exp Dermatol. 2006;15:678-84.

46. Sticozzi C, Cervellati F, Muresan XM, Cervellati C, Valacchi G. Resveratrol prevents smoke-induced keratinocytes damage. Food Funct. 2014;5:2348-56.

47. Timmers S, Konings E, Bilet L, Houtkooper RH, van de Weijer T, Goossens GH, et al. Calorie restriction-like effects of 30 days of resveratrol supplementation on energy metabolism and metabolic profile in obese humans. Cell Metab. 2011;14:612-22.

48. Shay KP, Moreau RF, Smith EJ, Smith AR, Hagen TM. Alphalipoic acid as a dietary supplement: Molecular mechanisms and therapeutic potential. Biochim Biophys Acta. 2009;1790:1149:60

49. Ptersen Shay K, Moreau RF, Smith EJ, Hagen TM. Is alphalipoic acid a scavenger of reactive oxygen species in vivo? Evidence for its initiation of stress signaling pathways that promote endogenous antioxidant capacity. IUBMB Life. 2008;60:362-7.

50. Merry BJ, Kirk AJ, Goyns MH. Dietary lipoic acid supplementation can mimic or block the effect of dietary restriction on life span. Mech Ageing Dev. 2008;129:341-8.

51. Javed S, Petropoulos IN, Alam U, Malik RA. Treatment of painful diabetic neuropathy. Ther Adv Chronic Dis. 2015;6:15-28

52. Papanas N, Ziegler D. Efficacy of $\alpha$-lipoic acid in diabetic neuropathy. Expert Opin Pharmacother. 2014;15:2721-31.

53. Gidal BE. New and emerging treatment options for neuropathic pain. Am J Manag Care. 2006;12(Suppl.):S269-78.

54. Shindo Y, Witt E, Han D, Epstein W, Packer L. Enzymic and non-enzymic antioxidants in epidermis and dermis of human skin. J Invest Dermatol. 1994;102:122-4.

55. Rhie G, Shin MH, Seo JY, Choi WW, Cho KH, Kim KH, et al. Aging-and photoaging dependent changes of enzymic and nonenzymic antioxidants in the epidermis and dermis of human skin in vivo. J Invest Dermatol. 2001;117:1212-7.

56. Hernández-Pérez E, Khawaja HA, Álvarez TYM. Oral isotretinoin as part of the treatment of cutaneous aging. Dermatol Surg. 2000;26:649-52.

57. Rabello-Fonseca RM, Azulay DR, Luiz RR, MandarimdeLacerda CA, Cuzzi T, Manela-Azulay M. Oral isotretinoin in photoaging: Clinical and histopathological evidence of efficacy of an off-label indication. J Eur Acad Dermatol Venereol. 2009;23:115-23.

58. Kalil CL, Fachinello FZ, Lamb FM, Comunello LN. Use of ora isotretinoin in photoaging therapy. Skinmed. 2008;7:10-4.

59. Anstey AV. Systemic photoprotection with a-tocopherol (vitamin E) and ß-carotene. Clin Exp Dermatol. 2002;27:170-6.

6o. Eicker J, Kurten V, Wild S, Riss G, Goralczyk R, Krutmann J, et al. Betacarotene supplementation protects from photoaging-associated DNA mutation. Photochem Photobiol Sci. 2003;2:655-9.

61. McArdle F, Rhodes LE, Parslew RA, Close GL, Jack CI, Friedmann PS, et al. Effects of oral vitamin E and B-carotene supplementation on ultraviolet radiation induced oxidative stress in human skin. Am J Clin Nutr. 2004;80:1270-5. 
62. Richelle M, Sabatier M, Steiling H, Williamson G. Skin bioavailability of dietary vitamin E, carotenoids, polyphenols, vitamin C, zinc, and selenium. Br J Nutr. 2006;96:227-38.

63. Garmyn M, Ribaya-Mercado JD, Russel RM, Bhawan J, Gilchrest BA. Effect of beta-carotene supplementation on the human sunburn reaction. Exp Dermatol. 1995;4:104-11.

64. Eberlein-Konig B, Placzek M, Przybilla B. Protective effect against sunburn of combined systemic ascorbic acid (vitamin C) and d-alpha-tocopherol (vitamin E). J Am Acad Dermatol. 1998;38:45-8.

65. Fuchs J, Kern H. Modulation of UV-light-induced skin inflammation by d-a-tocopherol and L-ascorbic acid: A clinical study using solar simulated radiation. Free Radic Biol Med. 1998;25:1006-12.

66. González S, Gilaberte Y, Philips, Juarranz A. Fernblock, a nutriceutical with photoprotective properties and potential preventive agent for skin photoaging and photoinduced skin cancers. Int J Mol Sci. 2011;12:8466-75.

67. El-Haj N, Golstein N. Sun protection in a pill: The photoprotective properties of Polypodium leucotomos extract. Int J Dermatol. 2015;54:362-6.

68. González S, Gilaberte Y, Philips N. Current Trends in photoprotection - a new generation of oral photoprotectors. The Open Dermatology Journal. 2011;5:1-9.

69. Bafana A, Dutt S, Kumar A, Kumar S, Ahuja PS. The basic and applied aspects of superoxide dismutase. Journal of Molecular Catalysis B: Enzimatic. 2010;68:129-38.

70. Nakajima S, Ohsawa I, Nagata K, Ohta S, Ohno M, Ijichi $\mathrm{T}$, et al. Oral supplementation with melon superoxide dismutase extract promotes antioxidant defenses in the brain and prevents stress-induced impairment of spatial memory. Behav Brain Res. 2009;200:15-21

71. Skarpanska-Stejnborn A, Pilaczynska-Szczesniak L, Basta P. Effects of oral supplementation with plant superoxide dismutase extract on selected redox parameters and an inflammatory marker in a 2,00o-m rowing-ergometer test. Int J Sport Nutr Exerc Metab. 2011;21:124-34. 